\title{
Raman and X-Ray photoelectron spectroscopic studies of graphene devices for identification of doping
}

\author{
Pinar Aydogan Gokturk ${ }^{a}$, Nurbek Kakenov ${ }^{b}$, Coskun Kocabas ${ }^{b}$, Sefik Suzera,* \\ a Department of Chemistry, Bilkent University, Bilkent, 06800, Ankara, Turkey \\ ${ }^{\mathrm{b}}$ Department of Physics, Bilkent University, Bilkent, 06800, Ankara, Turkey
}

\section{A R T I C L E I N F O}

\section{Article history:}

Received 25 April 2017

Received in revised form 5 July 2017

Accepted 13 July 2017

Available online 18 July 2017

\section{Keywords:}

X-Ray photoelectron spectroscopy (XPS)

Graphene

Polymer induced doping

Raman spectroscopy

Work-function

\begin{abstract}
A B S T R A C T
Tunability of electronic properties of graphene is one of the most promising properties to integrate it to high efficiency devices in the field of electronics. Here we demonstrate the substrate induced doping of CVD graphene devices using polymers with different functional groups. Both X-Ray secondary electron cut-off and Raman spectra confirm $p$-type doping of a PVC-Graphene film when compared to a PMMAGraphene one. We also systematically analyzed the reversible doping effect of acid-base exposure and UV illumination to further dope/undope the polymer supported graphene devices. The shifts in the Raman 2D band towards lower and then to higher wavenumbers, with sequential exposure to ammonia and hydrochloric acid vapors, suggest $n$-type doing and restoration of graphene to its original state. Finally, the $n$-type doping with UV irradiation on half-covered samples was utilized and shown by both XPS and Raman to create two regions with different electronic properties and resistances. These type of controlled and reversible doping routes offer new paths for electronic devices especially towards fabricating graphene $p$ - $n$ junctions.
\end{abstract}

(c) 2017 Elsevier B.V. All rights reserved.

\section{Introduction}

Graphene, the two dimensional $\mathrm{sp}^{2}$ hybridized hexagonal honeycomb shaped network of pure carbon atoms, has received tremendous interest from researchers due to its unique properties especially in the field of electronics [1-5]. This can mostly be attributed to the tunability of electronic properties of graphene. One of the most widely used way for tuning the electronic properties is doping [6-12]. Graphene doping can be made by electrical, chemical, contact or optical means. In our previous study, we showed that applying a gate voltage, hence creating an electric field, induces free charge carriers on graphene and shifts the Fermi energy, which can be monitored by gate tunable photoemission of XPS peaks [13]. Contact doping is similar, but in this case the electric field is formed due to differences in the work functions of graphene and contact materials [14,15]. Many others use chemical doping $[6,8,16]$, which is generally relied on substitution of some of heteroatoms [17-19] to the carbon network or by surface transfer doping $[20,21]$. Although the substitutional doping results in doped and stable graphene, the $\mathrm{sp}^{2}$ carbon network is irreversibly disrupted due to defect formation, which also leads

\footnotetext{
* Corresponding author.

E-mail address: suzer@fen.bilkent.edu.tr(S. Suzer).
}

to decrease in conductivity and mobility. Chemical doping can also be achieved by exposing the graphene samples to gases or liquids which adsorb on the surface hence cause changes in the Fermi Energy [22]. Additionally, ultraviolet irradiation modulated graphene doping is also reported by many others [9,11,12,23-25]. Optically driven molecular structure transformation of adsorbed species that cause reversible doping of graphene has recently been reported using pyrene [7], spiropyran [25], and azo-based chromophores [9,12]. Luo et al., showed that a reversible and controlled n-type doping was caused by UV illumination of CVD graphene [11] while deep UV illumination under oxygen or ozone environment was reported to induce $p$-type doping $[23,24]$.

Raman spectroscopy is common and well established technique for analysis of graphene and its derivatives. By this method, not only the quality of the layer can be assessed, but also the number of layers of graphene materials can be identified [26-28]. For graphene, Raman spectra show two main features which are the so-called G and $2 \mathrm{D}$ bands, and the position of these bands are used to determine the type of doping [29,30]. Additionally, presence of D and D' bands in the Raman spectrum, which are also called defect activated bands, indicative of the changes in the $\mathrm{sp}^{2}$ hybridized structure of graphene, have also been used to probe the presence of impurities or defects $[28,31,32]$.

$\mathrm{X}$-Ray photoelectron spectroscopy is also frequently used for elemental analysis and also for elucidating the nature of the doped 


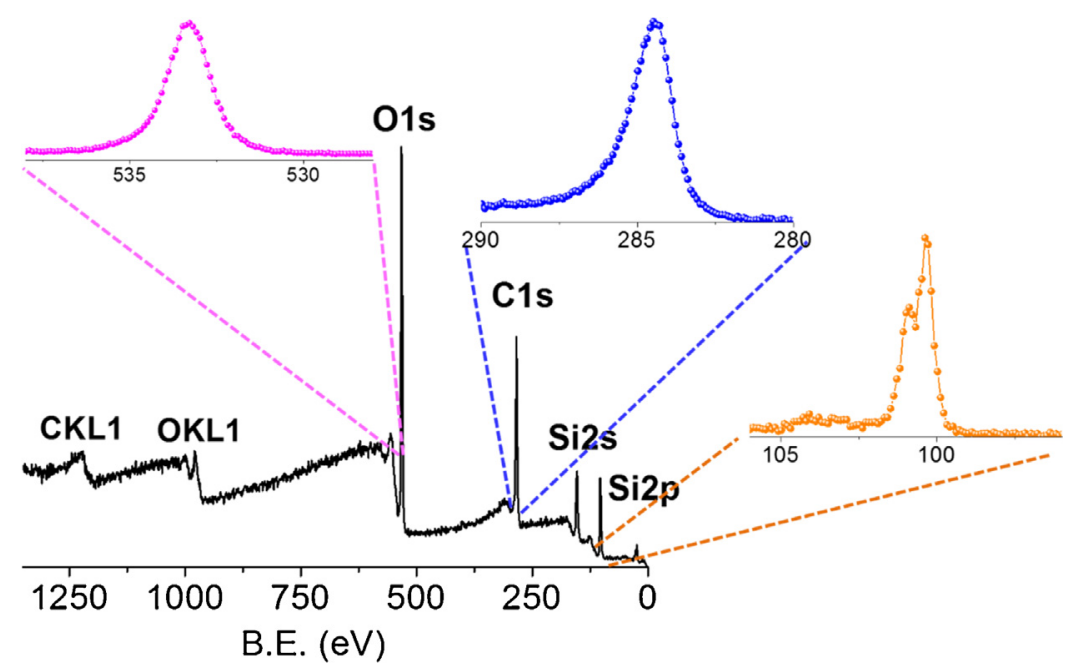

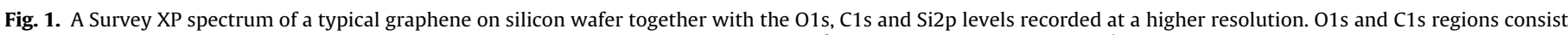
of a single peak whereas $\mathrm{Si} 2 \mathrm{p}$ consists of two peaks, assignable to lower binding energy $\mathrm{Si}^{0}$ and higher binding energy $\mathrm{Si}^{+4}$.

heteroatoms $[10,17,33]$. In XPS, the sample is irradiated by a known energy of X-rays which results in creation of photoelectrons whose kinetic energies are determined and converted to binding energies using the energy of the X-ray photons and the spectrometer work function. Binding energy of a specific level is different for each atom, which also varies with its chemical environment. Therefore, XPS gives chemical as well as elemental information. XP Survey and high resolution $\mathrm{Si2p}, \mathrm{C} 1 \mathrm{~s}$ and $01 \mathrm{~s}$ spectra for a typical sample of graphene on $\mathrm{SiO}_{2} / \mathrm{Si}$ substrate are shown in Fig. 1. Since XPS is a surface sensitive technique and give information only about the top few nanometers depth of the sample's surface, and graphene is a single (or a few) layer of carbon atoms, silicon substrate is within the analysis depth and is observable both in the survey and in the high resolution (in the energy scale) scans. For example, while the $\mathrm{Si} 2 \mathrm{p}_{1 / 2}$ peak appears around $100 \mathrm{eV}$ for $\mathrm{Si}^{0}$ we also observe a small intensity peak around $104 \mathrm{eV}$ corresponding to the $\mathrm{Si}^{4+}$ of the thin native oxide $\left(\mathrm{SiO}_{2}\right)$ layer. In conventional usage, XPS is used to harvest information on; (i) a selected point, (ii) along a line (line-scan), or (iii) on the entire area (areal scan) with a lateral resolution of $30-400 \mu \mathrm{m}$. However, in all the data reported in this work, only $100 \mu \mathrm{m}$ spot and $100 \mu \mathrm{m}$ step sizes are used. In line scan or areal modes, since the number of spectra are large, the spectral information is usually condensed and displayed as variations either in; (i) the intensity or (ii) the binding energy positions of the selected regions.

In one alternative use of XPS, application of external bias to the sample is used to cause development of additional charges on the surface, which allows the user to extract additional electrical properties about the sample, because the position of photoelectron peaks also shifts under the applied potential. For example, the binding energy of $\mathrm{Au}_{4 / 2}$ peak is $84.0 \mathrm{eV}$, corresponding to the zero oxidation state gold atoms within the metal. When we apply an external bias to the gold substrate the position of photoelectron peaks shifts with the same amplitude as the applied bias due to its excellent conductivity, see Fig. 2. However, shifts with different magnitudes are usually observed for poorly-conductive or non-conductive materials.

In one of our previous work, we showed that variations in the electrical potential, derived from the positions of the $\mathrm{C} 1 \mathrm{~s}$ binding energy throughout the sample due to external bias are linearly correlated with the bias applied for a pristine graphene [34]. However, by analysis of peak positions, it was also demonstrated that the external bias reflects and amplifies the appearance of morphological defects on the graphene layer, see Fig. 3b [35].

As we discussed earlier in this paper, XPS is a charge sensitive technique so that the peak positions are also affected and shift due to charging on the surface. This makes XPS a good candidate to analyze doping in theory. However, as we had previously shown that the energy difference between the $p$-type and $n$-type Si2p peaks on $\mathrm{H}$ terminated $\mathrm{Si}$ wafer surfaces is much smaller $(0.18 \mathrm{eV})$ due to band bending, hence it is difficult to fully evaluate the effect of doping, as shown in Fig. 4.

Another possibility that photoelectron spectroscopy offers is the determination of the work function of the sample through analysis of the low energy cut-off region, dominated by secondary electrons. Such determinations are also used for assessing the type and the extent of doping. The onset of the secondary electron cut-off gives information about the work function of the sample using the relation;

$\phi_{\text {sample }}=h v-\left(E_{K, \max }-E_{K, \min }\right)$

where $E_{K, \max }$ is the maximum measured kinetic energy of an electron emitted from the Fermi level, and $E_{K, \min }$ is the minimum measured kinetic energy which is located in the region of the secondary electron cut-off [36,37]. Even though, UPS is generally used for work function determinations due to its higher photon flux $[38,39]$, XPS has also been used in the literature [37,40,41]. However, experimentally it is challenging to measure the electrons with nearly zero kinetic energy using a conventional XPS spectrometer. That is why, generally a negative bias is applied to detect and analyze this region, because the negative bias accelarates the kinetic energy of all electrons to the spectrometer. In our case, to analyze systematically the secondary cut-off region a $-30 \mathrm{~V}$ external bias is applied from the bottom of the silicon wafers. Since this applied bias gives an offset to the entire energy scale in the spectrum, the above equation is still valid. The high resolution Si2p and secondary electron cut-off region are shown in Fig. 4 for both an $n$-type and a p-type H-terminated Si wafers which shows a $\sim 0.30 \mathrm{eV}$ difference in the cut-off edge, as opposed to $0.18 \mathrm{eV}$ difference in the Si2p peak.

Mainly for the electronic applications of graphene the biggest limitation is the absence of a semiconducting band gap. For this reason, many researchers have investigated the doping of graphene which is an effective method to tune its electronic property. This 


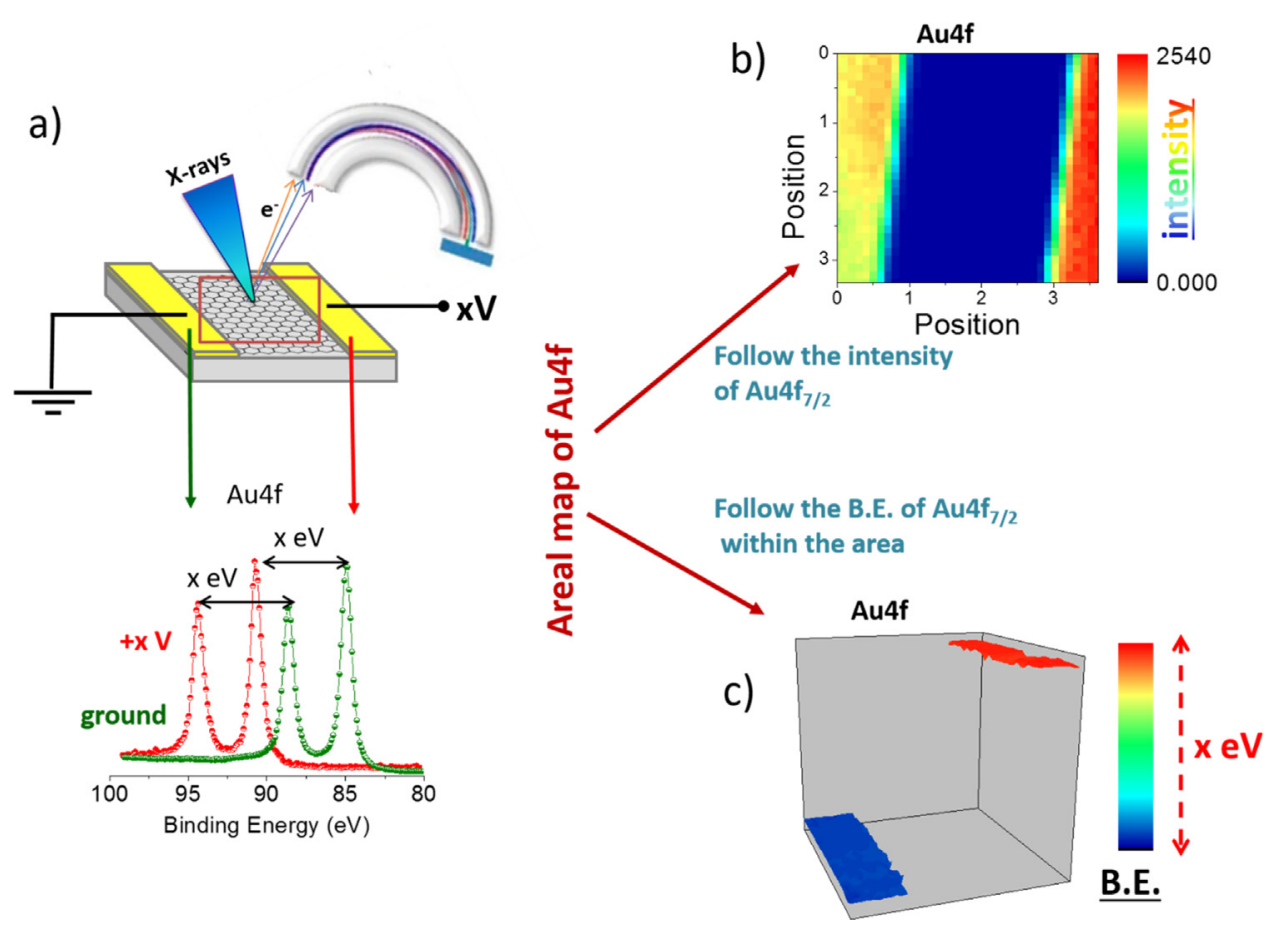

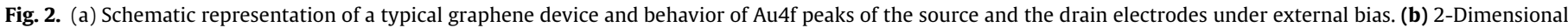

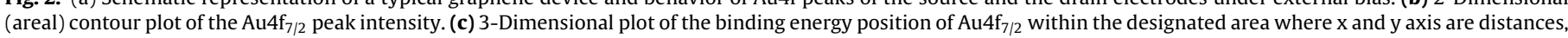
while the $\mathrm{z}$ and color bar both represents the binding energy scale. Data is recorded with $100 \mu \mathrm{m}$ of X-rays spot size and with $100 \mu \mathrm{m}$ steps in between two points.

a)

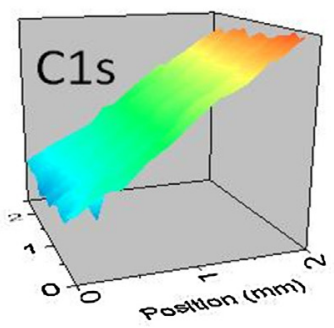

std.dev $=0.11$

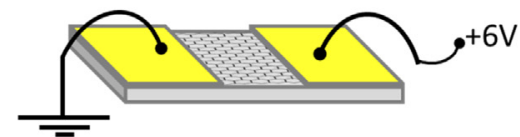

Under external bias $330 \mathrm{Ohm}$ pristine graphene b)

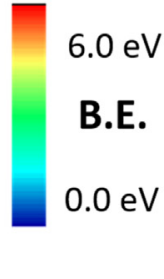

B.E.

eV

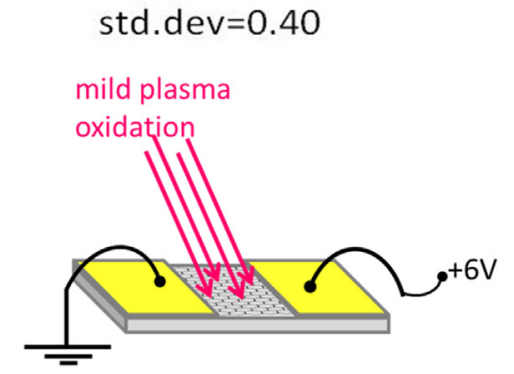

Under external bias $4 \mathrm{kOhm}$
defective graphene

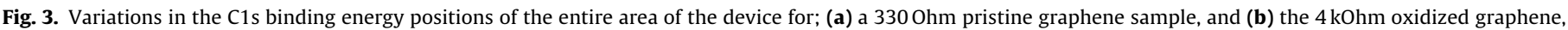
under $+6 \mathrm{~V}$ external bias to one of the electrodes, while the other is grounded. Their schematic representations are given at the bottom.

work presents a controlled doping of flexible graphene devices achieved by various doping methods and brings a new perspective to characterize it. In this work, by using XPS and Raman Spectroscopy as complementary techniques we investigated substrate induced doping of two polymers with different functional groups on CVD graphene layers. In addition, we used acid and base exposure and also ultraviolet illumination in order to create further doping. Fabrication of a device with two regions, each having a different type of doping, is also demonstrated.

\section{Experimental}

\subsection{Device preparation}

The graphene used to from devices is produced on a copper foil by chemical vapor deposition at $1035^{\circ} \mathrm{C}$ and 10 Torr pressure using flowing methane and hydrogen gases. 2\% solutions of PMMA and PVC were prepared in acetone and THF respectively. After the graphene growth, PMMA and PVC solutions are deposited on the graphene side by drop casting. After the coating, 


\section{a)}

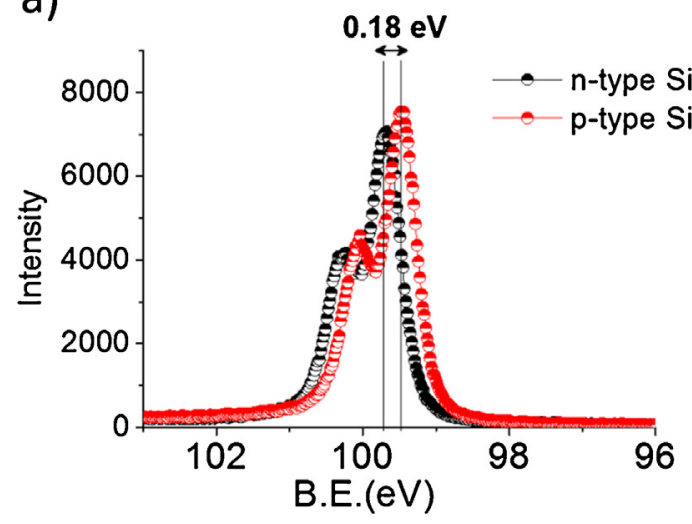

b)

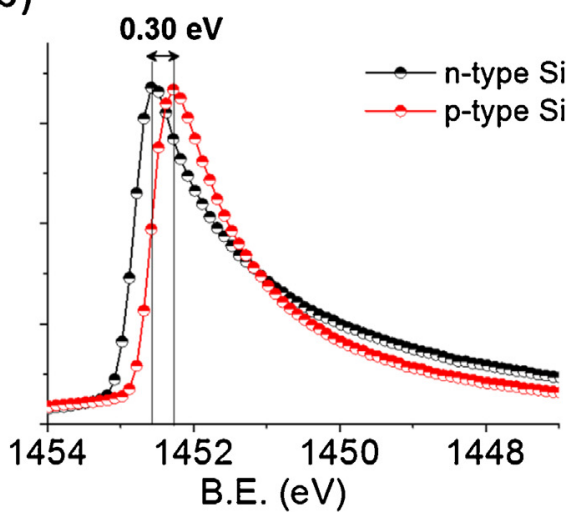

Fig. 4. XP spectra of (a) Si2p and (b) Cut-Off regions recorded of a $p$-type and an $n$-type H-terminated Si samples.

a)

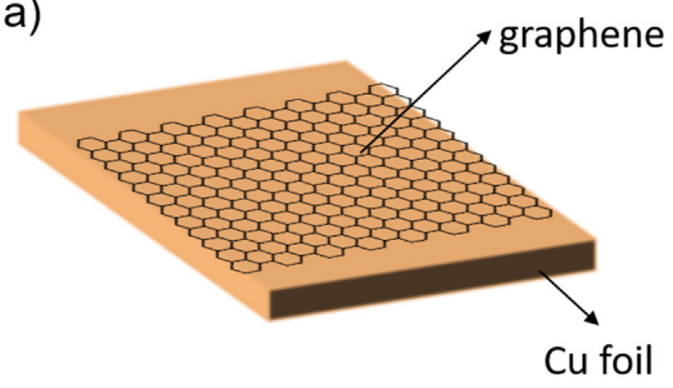

b)

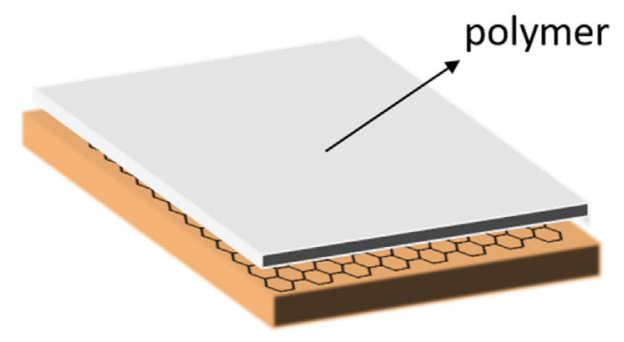

d)

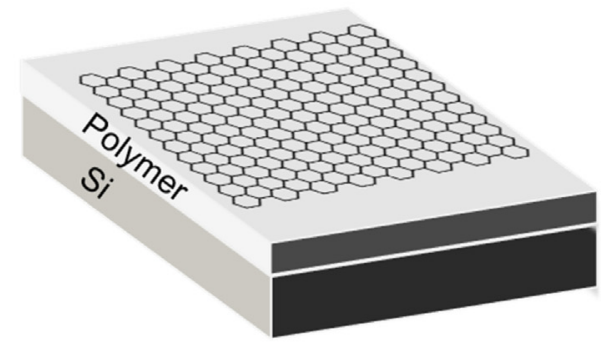

c)

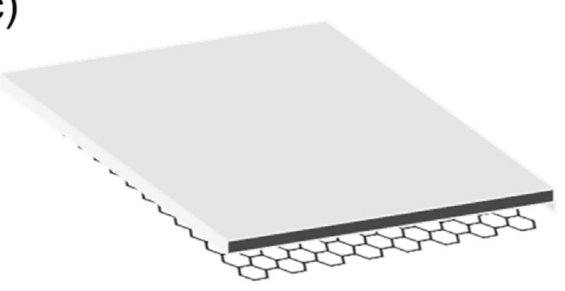



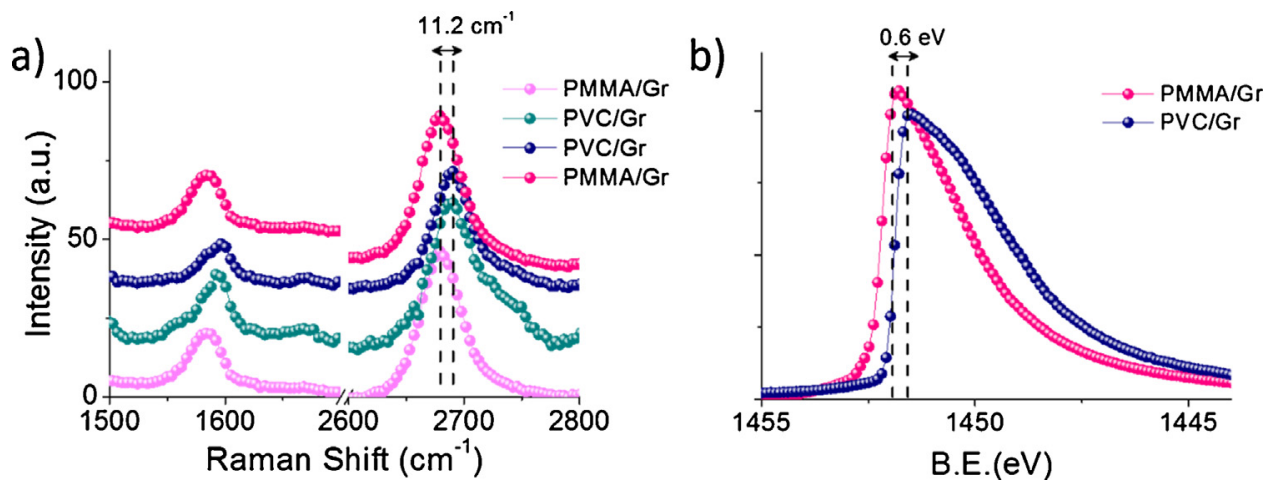

Fig. 6. (a) Raman and (b) XP Cut-Off spectra of the PVC/Graphene and the PMMA/Graphene films.
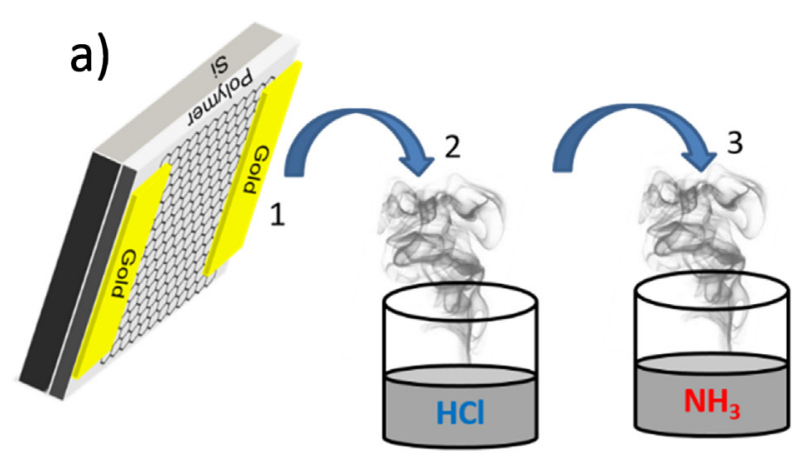

c)

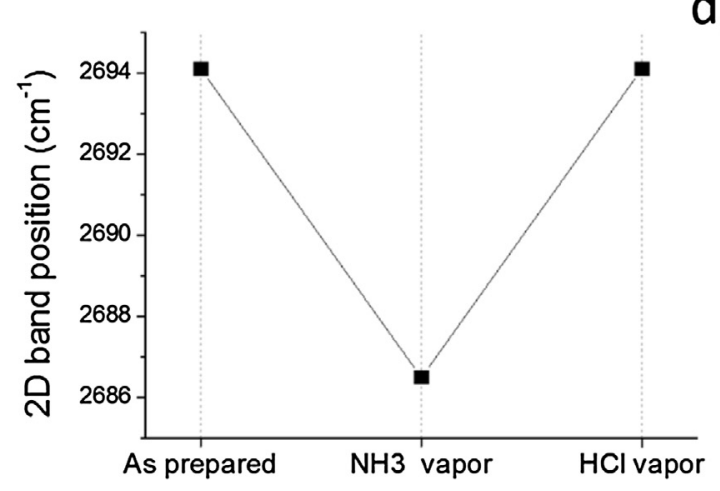

b)

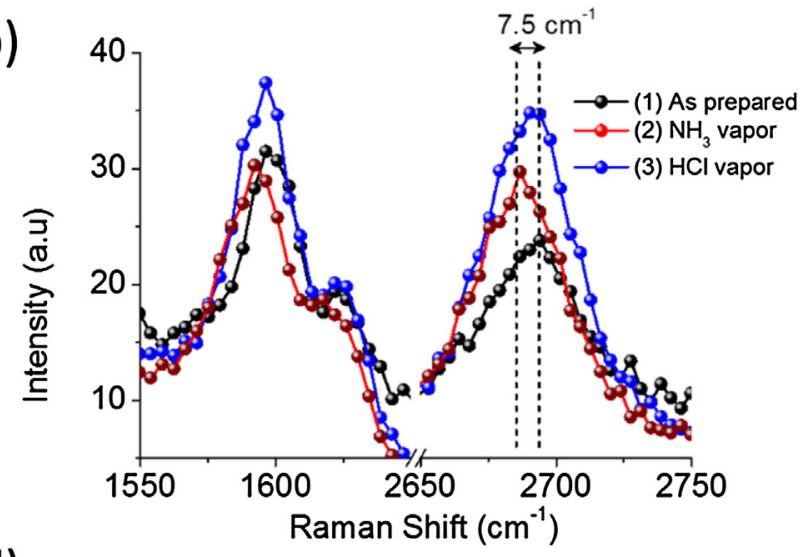

)

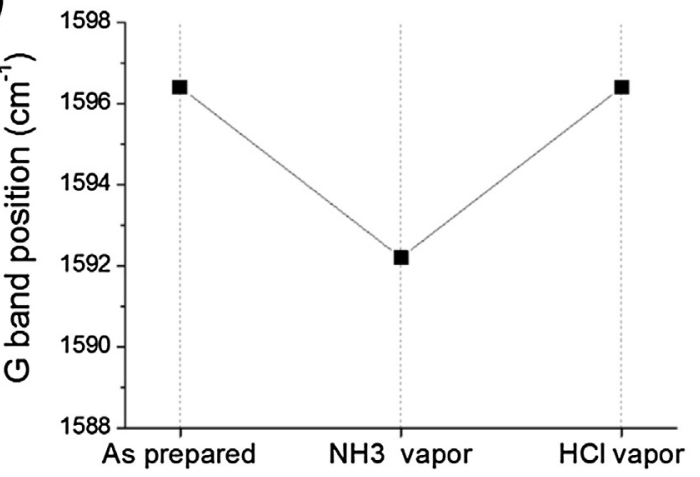

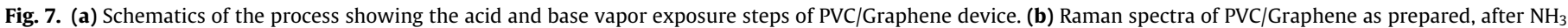
and then $\mathrm{HCl}$ exposure. (c) Shift of Raman 2D band and (d) $\mathrm{G}$ band position between treatments for as prepared, after exposure to $\mathrm{HCl}$ vapor, and then to $\mathrm{NH}_{3}$ vapor.

electrodes are connected to the sample holder in order to apply a $-30 \mathrm{~V}$ bias to the entire holder. On the other hand, for the Voltage Contrast XPS measurements one of the electrodes is grounded while the external bias is applied to the other one, inducing current flow across the device. Raman spectra are obtained with a Jobin Yvon LABRAM Raman Spectrometer equipped with $532 \mathrm{~nm}$ green laser. The laser beam is focused by a $50 \mathrm{x}$ objective lens. The scattered radiation is collected by the same objective lens and sent to a CCD detector.

\section{Results and discussion}

\subsection{Substrate induced effects}

Raman spectra for PMMA and PVC supported graphene are shown in Fig. 6a. Since the characteristic fingerprints of PVC and PMMA appear in the region below $1500 \mathrm{~cm}^{-1}$ we only focused on the $G$ and 2D peaks coming from the graphene layer where $G$ band is due to Raman active doubly degenerate in plane stretching of sp2 carbons corresponding to $\mathrm{E}_{2 \mathrm{~g}}$ mode, and 2D is an overtone of $\mathrm{D}$ band which is the in-plane breathing-like mode of carbon rings [26,28]. The position of these two features gives information about doping. An upshift of the $G$ band usually demonstrates the occurrence of doping (holes or electrons) in graphene films, while the shift in 2D indicates the type of doping. Downshift in 2D band indicates an $n$-type and upshift indicates a p-type doping [29,42]. It is also wellknown that, molecules with electron withdrawing groups adsorbed on the surface of graphene will lead to $p$-type doping of graphene, and molecules with electron donating groups will lead to $n$-type doping $[7,43,44]$. In this case, since both the $G$ and the $2 \mathrm{D}$ bands shift to higher wavenumbers, the PVC-Graphene film exhibits $p$ type shifting compare to the PMMA-Graphene film. As can be seen a shift of $\sim 11 \mathrm{~cm}^{-1}$ is observed, which is reproducible. In the recent work of Srivastava Luo et al. [49], doping of monolayer graphene 
a)
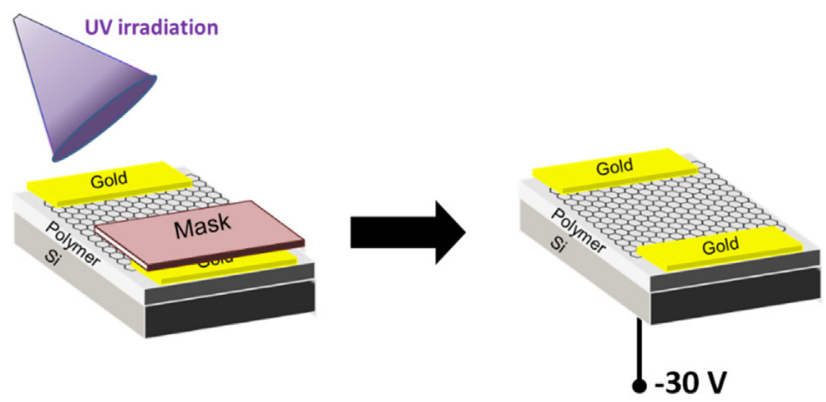

b)

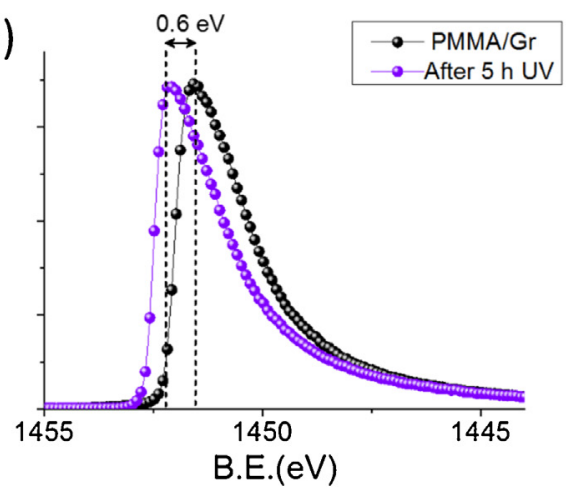

c)

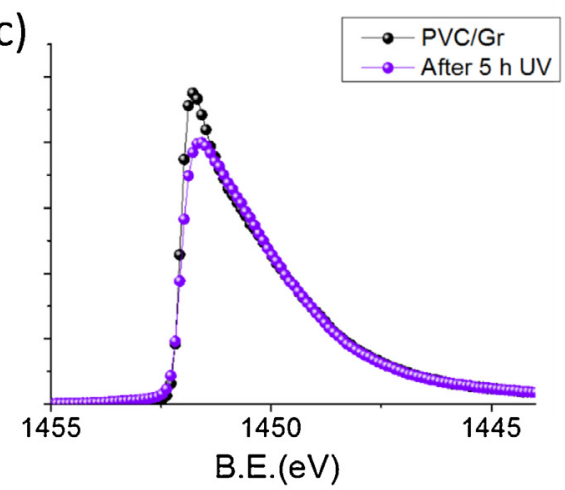

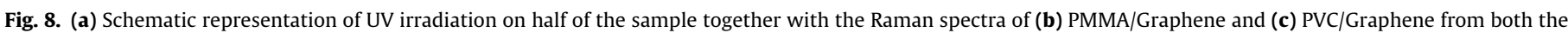
masked and UV-irradiated regions.

through the charge transfer by the trapping organic molecules such as toluene, chlorobenzene, acetone, DMF, and PC was reported. In order to check any effect coming from the solvent that we used during the sample preparation, we diped PMMA/Gr sample to the THF and PVC/Gr sample to the acetone and checked the Raman Spectra which does not show any peak shifting on the $G$ and 2D bands, see Supporting Information.

As was shown in Fig. 4 for $n$ - and $p$-doped Si wafers, the work function is sensitive to the doping level and can be probed by the secondary electron cut-off measurements under $-30 \mathrm{~V}$ bias. Similar behavior can also be observed for the polymer supported graphene samples, which are now shown in Fig. 6b, together with the corresponding shifts detected through Raman measurements shown in Fig. 6a. Hence, the secondary electron cut-off measurements also support the more $p$-type behavior of the graphene on PVC, compared to the graphene on PMMA, which has $\sim 0.6 \mathrm{eV}$ higher cut-off energy. Note also that more than three different samples are prepared and analyzed for ensuring the reproducibility of these findings.

\subsection{Reversible doping with acid/base exposure}

Having demonstrated that the substrates do induce controllable and stable doping on the CVD graphene, we now turn to investigate the effect of exposing the graphene-PVC sample to vapors of acid and base solutions. In this type of doping the exposed molecules are adsorbed onto the surface and act like acceptors or donors. While a variety of acids and bases have been used in literature, nitric acid is the commonly used one, which is known to reproducibly cause p-type doing on the pristine graphene samples $[45,46]$. However, to our knowledge, doping by acid/base vapors as a further doping/undoping of the already doped samples has not been reported before. To check the applicability on the graphene samples in the presence of a polymer as a substrate, we sequentially exposed the sample to the vapors of ammonia and then hydrochloric acid and recorded quickly the Raman spectra after each step. Raman spectra of the graphene sample on PVC; (i) as prepared, (ii) after $\mathrm{NH}_{3}$, and (iii) $\mathrm{HCl}$ exposure are shown in Fig. 7b, and the shifts in the $\mathrm{G}$ and the 2D bands are depicted in Fig. 7c.

Since the changes in the $G$ band position do not reveal information about the type of the induced doping, it is more informative to evaluate the 2D shifts. Exposing the graphene on PVC to the ammonia vapors causes a blue shift in the spectrum hence indicates induced $n$-doping which can be reversed back with further exposure to $\mathrm{HCl}$. We also observe that this type of doping is reversible and turns to its original state after a few hours. That is the reason why we do not show the secondary electron cut-off spectra using XPS, which is performed under ultra-high vacuum conditions, and after prolonged pump-times.

\subsection{UV irradiation and voltage contrast XPS}

Although UV irradiation is extensively used to induce doping in different ways in the literature, from our previous studies we know that UV also affects the polymer substrate. For example, in References [47] and [48], we had shown that UV irradiation of PVC causes photo-degradation and releases $\mathrm{HCl}$, i.e. acid vapors, which we also expect to effect the Fermi energy of the graphene overlayer. For this reason, we irradiated only half of both types of polymer supported graphene samples for a duration of $5 \mathrm{~h}$ with UV lamp (mostly $254 \mathrm{~nm} \mathrm{Hg}$-Line) by masking the other half. This type of UV irradiation reduces optical transparency of the samples and blocks the Raman measurement by inducing a saturation in the Raman signal. Therefore, only the secondary electron cut-off measurements under $-30 \mathrm{~V}$ on the UV irradiated and masked region are shown in Fig. 8b and 8c for PMMA and PVC supported CVD graphene films, respectively. While the cut-off edge clearly shows the $n$-type doping upon the UV irradiation on PMMA supported sample which is also consistent with the previous study of Luo et al., who showed the reversible and controlled $n$-type doping by UV illumination of 

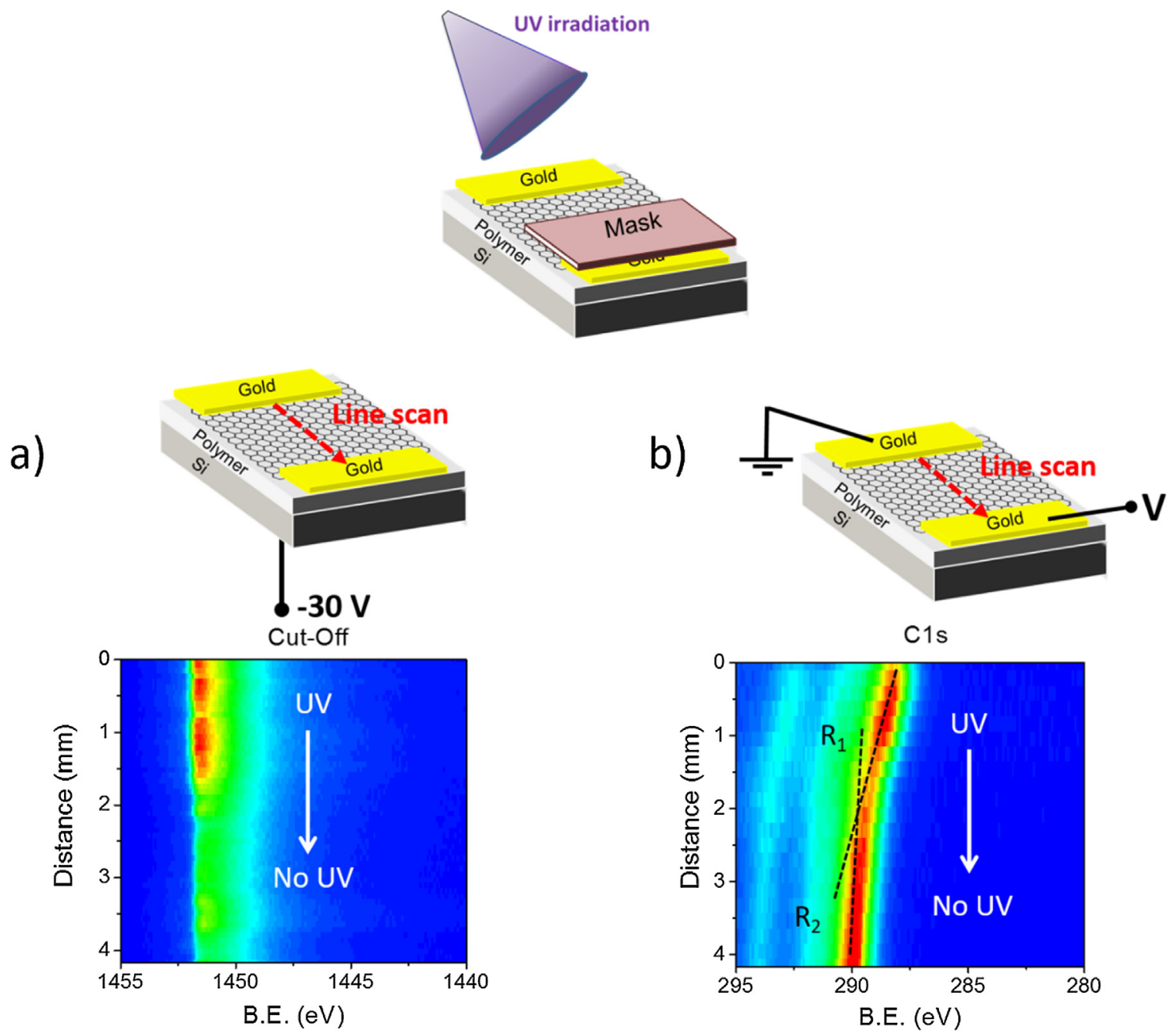

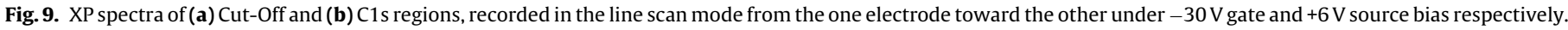

CVD graphene [11], there is no measurable shift (hence doping effect) induced by the UV irradiation on the PVC supported one. We believed that the main reason is the near cancelation of the two opposing effects; (i) $n$-type doping of the UV, and (ii) $p$-type doping of the $\mathrm{HCl}$ formation during the irradiation.

For additional information, we recorded the secondary electron cut-off spectra on a line starting from the UV irradiated towards the masked region of the PMMA supported graphene sample, where the clear indication of two regions with different work functions are observed as shown in Fig. 9a. Furthermore, we fabricated a device on the same sample by depositing gold electrodes in the source-drain geometry and applied an external bias from one end while the other one is grounded, and acquired spectra on a similar line as in Fig. 9a, which are depicted in Fig. 9b. This type of measurements is called Voltage Contrast XPS and has extensively been used by our group [35]. For example, as we showed earlier in Fig. 3a that;(i) for the defect free pristine graphene sample the $\mathrm{C} 1 \mathrm{~s}$ binding energy drop was linear along the line (IR drop), and (ii) in the presence of defects this drop deviated strongly from linearity [22]. However, very different from the previous findings is that, for the case of half UV irradiated PMMA supported graphene, two different regions with two different slopes are observed, indicating that the two regions have different resistances and carrier concentrations, probed by both the cut-off and also the charge-contrast measurements.

\section{Conclusions}

A simple and easy method for doping of CVD-grown graphene is performed using polymer substrates having different functional groups. This method can be applied to any polymer which is not soluble in water. The functional groups of the underlying polymer layer induce doping in the graphene layer. The doping characteristics analyzed for two types of polymer, PMMA and PVC, by Raman Spectroscopy and XPS. This method causes the permanent doping on the graphene layers without introducing further defects. However, by controlled exposure to acid/base vapors or UV one can create regions with different doping types and/or levels in the same film, which might even lead to fabrication of $p$ - $n$ junctions, electrical properties of which can easily be assessed by the introduced simple variants of XPS measurements.

\section{Acknowledgements}

This work was partially supported by the Scientific and Technological Research Council of Turkey (TUBITAK) [grant number 215Z534] and C.K. also acknowledges the support from the European Research Council (ERC) Consolidator [Grant No ERC. 682723 Smart Graphene]. 


\section{Appendix A. Supplementary data}

Supplementary data associated with this article can be found, in the online version, at http://dx.doi.org/10.1016/j.apsusc.2017.07. 097.

\section{References}

[1] A.H. Castro Neto, F. Guinea, N.M.R. Peres, K.S. Novoselov, A.K. Geim, The electronic properties of graphene, Rev. Mod. Phys. 81 (2009) 109-162.

[2] K.S. Novoselov, A.K. Geim, S.V. Morozov, D. Jiang, Y. Zhang, S.V. Dubonos, I.V. Grigorieva, A.A. Firsov, Electric field effect in atomically thin carbon films, Science 306 (2004) 666-669.

[3] N.M.R. Peres, F. Guinea, A.H. Castro Neto, Electronic properties of disordered two-dimensional carbon, Phys. Rev. B 73 (2006) 125411

[4] S. Wang, P.K. Ang, Z. Wang, A.L.L. Tang, J.T.L. Thong, K.P. Loh, High mobility, printable, and solution-Processed graphene electronics, Nano Lett. 10 (2010) 92-98.

[5] Y. Zhang, Y.-W. Tan, H.L. Stormer, P. Kim, Experimental observation of the quantum Hall effect and Berry's phase in graphene, Nature 438 (2005) 201-204.

[6] F. Güneș, H.-J. Shin, C. Biswas, G.H. Han, E.S. Kim, S.J. Chae, J.-Y. Choi, Y.H. Lee, Layer-by-Layer doping of few-Layer graphene film, ACS Nano 4 (2010) 4595-4600.

[7] X. Dong, D. Fu, W. Fang, Y. Shi, P. Chen, L.-J. Li, Doping single-Layer graphene with aromatic molecules, Small 5 (2009) 1422-1426.

[8] D.B. Farmer, Y.-M. Lin, A. Afzali-Ardakani, P. Avouris, Behavior of a chemically doped graphene junction, Appl. Phys. Lett. 94 (2009) 213106.

[9] M. Kim, N.S. Safron, C. Huang, M.S. Arnold, P. Gopalan, Light-Driven reversible modulation of doping in graphene, Nano Lett. 12 (2012) 182-187.

[10] X. Li, H. Wang, J.T. Robinson, H. Sanchez, G. Diankov, H. Dai, Simultaneous nitrogen doping and reduction of graphene oxide, J. Am. Chem. Soc. 131 (2009) 15939-15944.

[11] Z. Luo, N.J. Pinto, Y. Davila, A.T.C. Johnson, Controlled doping of graphene using ultraviolet irradiation, Appl. Phys. Lett. 100 (2012) 253108

[12] N. Peimyoo, J. Li, J. Shang, X. Shen, C. Qiu, L. Xie, W. Huang, T. Yu, Photocontrolled molecular structural transition and doping in graphene, ACS Nano 6 (2012) 8878-8886.

[13] M. Copuroglu, P. Aydogan, E.O. Polat, C. Kocabas, S. Süzer, Gate-Tunable photoemission from graphene transistors, Nano Lett. 14 (2014) 2837-2842.

[14] G. Giovannetti, P.A. Khomyakov, G. Brocks, V.M. Karpan, J. van den Brink, P.J. Kelly, Doping graphene with metal contacts, Phys. Rev. Lett. 101 (2008) 026803.

[15] P.A. Khomyakov, G. Giovannetti, P.C. Rusu, G. Brocks, J. van den Brink, P.J. Kelly, First-principles study of the interaction and charge transfer between graphene and metals, Phys. Rev. B 79 (2009) 195425.

[16] H. Sojoudi, J. Baltazar, L. Tolbert, C. Henderson, S. Graham, Formation of air stable graphene $\mathrm{p}$ ?n?p junctions using an amine-Containing polymer coating, Adv. Mater. Interfaces 1 (2014) 1400378 (-n/a).

[17] D. Usachov, O. Vilkov, A. Grüneis, D. Haberer, A. Fedorov, V.K. Adamchuk, A.B. Preobrajenski, P. Dudin, A. Barinov, M. Oehzelt, C. Laubschat, D.V. Vyalikh, Nitrogen-Doped graphene: efficient growth, structure, and electronic properties, Nano Lett. 11 (2011) 5401-5407.

[18] L. Zhao, R. He, K.T. Rim, T. Schiros, K.S. Kim, H. Zhou, C. Gutiérrez, S.P. Chockalingam, C.J. Arguello, L. Pálová, D. Nordlund, M.S. Hybertsen, D.R. Reichman, T.F. Heinz, P. Kim, A. Pinczuk, G.W. Flynn, A.N. Pasupathy, Visualizing individual nitrogen dopants in monolayer graphene, Science 333 (2011) 999-1003.

[19] E.H. Åhlgren, J. Kotakoski, A.V. Krasheninnikov, Atomistic simulations of the implantation of low-energy boron and nitrogen ions into graphene, Phys. Rev. B 83 (2011) 115424

[20] Z. Chen, I. Santoso, R. Wang, L.F. Xie, H.Y. Mao, H. Huang, Y.Z. Wang, X.Y. Gao, Z.K. Chen, D. Ma, A.T.S. Wee, W. Chen, Surface transfer hole doping of epitaxial graphene using MoO3 thin film, Appl. Phys. Lett. 96 (2010) 213104.

[21] J. Meyer, P.R. Kidambi, B.C. Bayer, C. Weijtens, A. Kuhn, A. Centeno, A. Pesquera, A. Zurutuza, J. Robertson, S. Hofmann, Metal oxide induced charge transfer doping and band alignment of graphene electrodes for efficient organic light emitting diodes, Sci. Rep. 4 (2014) 5380.

[22] X. Wang, X. Li, L. Zhang, Y. Yoon, P.K. Weber, H. Wang, J. Guo, H. Dai, N-Doping of graphene through electrothermal reactions with ammonia, Science 324 (2009) 768-771

[23] S. Huh, J. Park, Y.S. Kim, K.S. Kim, B.H. Hong, J.-M. Nam, UV/Ozone-Oxidized large-Scale graphene platform with large chemical enhancement in surface-Enhanced raman scattering, ACS Nano 5 (2011) 9799-9806.

[24] M.Z. Iqbal, S. Siddique, M.W. Iqbal, J. Eom, Formation of p-n junction with stable p-doping in graphene field effect transistors using deep UV irradiation, J. Mater. Chem. C 1 (2013) 3078-3083
[25] P. Joo, B.J. Kim, E.K. Jeon, J.H. Cho, B.-S. Kim, Optical switching of the Dirac point in graphene multilayer field-effect transistors functionalized with spiropyran, Chem. Commun. 48 (2012) 10978-10980.

[26] A.C. Ferrari, Raman spectroscopy of graphene and graphite: disorder, electron-phonon coupling, doping and nonadiabatic effects, Solid State Commun. 143 (2007) 47-57.

[27] J. Ado, M.L. Marcia, S. Fernando, H.M.F. Erlon, V.O.M. Marcus, B.C. Rodrigo, A.A Carlos, Raman study of ion-induced defects in $\mathrm{N}$-layer graphene, J. Phys.: Condens. Matter 22 (2010) 334204

[28] A.C. Ferrari, J.C. Meyer, V. Scardaci, C. Casiraghi, M. Lazzeri, F. Mauri, S. Piscanec, D. Jiang, K.S. Novoselov, S. Roth, A.K. Geim, Raman spectrum of graphene and graphene layers, Phys. Rev. Lett. 97 (2006) 187401.

[29] PisanaS DasA, PiscanecS ChakrabortyB, S.K. Saha, U.V. Waghmare, K.S. Novoselov, H.R. Krishnamurthy, A.K. Geim, A.C. Ferrari, A.K. Sood, Monitoring dopants by Raman scattering in an electrochemically top-gated graphene transistor, Nat Nano 3 (2008) 210-215.

[30] J. Yan, Y. Zhang, P. Kim, A. Pinczuk, Electric field effect tuning of electron-Phonon coupling in graphene, Phys. Rev. Lett. 98 (2007) 166802.

[31] L.G. Cançado, A. Jorio, E.H.M. Ferreira, F. Stavale, C.A. Achete, R.B. Capaz, M.V.O. Moutinho, A. Lombardo, T.S. Kulmala, A.C. Ferrari, Quantifying defects in graphene via raman spectroscopy at different excitation energies, Nano Lett. 11 (2011) 3190-3196.

[32] M.M. Lucchese, F. Stavale, E.H.M. Ferreira, C. Vilani, M.V.O. Moutinho, R.B. Capaz, C.A. Achete, A. Jorio, Quantifying ion-induced defects and Raman relaxation length in graphene, Carbon 48 (2010) 1592-1597.

[33] Y. Shao, S. Zhang, M.H. Engelhard, G. Li, G. Shao, Y. Wang, J. Liu, I.A. Aksay, Y. Lin, Nitrogen-doped graphene and its electrochemical applications, J. Mater. Chem. 20 (2010) 7491-7496.

[34] C. Kocabas, S. Suzer, Probing voltage drop variations in graphene with photoelectron spectroscopy, Anal. Chem. 85 (2013) 4172-4177.

[35] P. Aydogan, E.O. Polat, C. Kocabas, S. Suzer, X-ray photoelectron spectroscopy for identification of morphological defects and disorders in graphene devices, J. Vac. Sci. Technol. A: Vac. Surf. Films 34 (2016) 041516

[36] A. Benayad, H.-J. Shin, H.K. Park, S.-M. Yoon, K.K. Kim, M.H. Jin, H.-K. Jeong, J.C. Lee, J.-Y. Choi, Y.H. Lee, Controlling work function of reduced graphite oxide with Au-ion concentration, Chem. Phys. Lett. 475 (2009) 91-95.

[37] M.G. Helander, M.T. Greiner, Z.B. Wang, Z.H. Lu, Pitfalls in measuring work function using photoelectron spectroscopy, Appl. Surf. Sci. 256 (2010) 2602-2605.

[38] J.-K. Chang, W.-H. Lin, J.-I. Taur, T.-H. Chen, G.-K. Liao, T.-W. Pi, M.-H. Chen, C.-I. Wu, Graphene anodes and cathodes tuning the work function of graphene by nearly $2 \mathrm{eV}$ with an aqueous intercalation process, ACS Appl. Mater. Interfaces 7 (2015) 17155-17161.

[39] S. Chandramohan, K. Ji Hye, Y.S. Katharria, H. Nam, B. Yun Seon, K. Kang Bok, P. Jong Bae, R. Beo Deul, K. Hyun Kyu, S. Eun-Kyung, H. Chang-Hee, Chemically modified multilayer graphene with metal interlayer as an efficient current spreading electrode for InGaN/GaN blue light-emitting diodes, J. Phys. D: Appl. Phys. 45 (2012) 145101.

[40] R. Schlaf, H. Murata, Z.H. Kafafi, Work function measurements on indium tin oxide films, J. Electron Spectrosc. Relat. Phenom. 120 (2001) 149-154.

[41] M.G. Helander, Z.B. Wang, J. Qiu, M.T. Greiner, D.P. Puzzo, Z.W. Liu, Z.H. Lu, Chlorinated indium tin oxide electrodes with high work function for organic device compatibility, Science 332 (2011) 944-947.

[42] C. Casiraghi, S. Pisana, K.S. Novoselov, A.K. Geim, A.C. Ferrari, Raman fingerprint of charged impurities in graphene, Appl. Phys. Lett. 91 (2007) 233108.

[43] H. Liu, Y. Liu, D. Zhu, Chemical doping of graphene, J. Mater. Chem. 21 (2011) $3335-3345$.

[44] C.N.R. Rao, A.K. Sood, K.S. Subrahmanyam, A. Govindaraj, Graphene the new two-Dimensional nanomaterial, Angew. Chem. Int. Ed. 48 (2009) 7752-7777.

[45] L. D'Arsie, S. Esconjauregui, R.S. Weatherup, X. Wu, W.E. Arter, H. Sugime, C. Cepek, J. Robertson, Stable, efficient p-type doping of graphene by nitric acid, RSC Adv. 6 (2016) 113185-113192.

[46] A. Kasry, M.A. Kuroda, G.J. Martyna, G.S. Tulevski, A.A. Bol, Chemical doping of large-Area stacked graphene films for use as transparent, conducting electrodes, ACS Nano 4 (2010) 3839-3844.

[47] U.A. Sevil, O. Güven, S. Süzer, Spectroscopic investigation of onset and enhancement of electrical conductivity in PVC/PANI composites and blends by $\gamma$-ray or UV irradiation, J. Phys. Chem. B 102 (1998) 3902-3905.

[48] O. Birer, S. Suzer, U.A. Sevil, O. Guven, UV-Vis IR and XPS analysis of UV induced changes in PVC composites, J. Mol. Struct. 482-483 (1999) 515-518.

[49] P.K. Srivastava, P. Yadav, V. Rani, S. Ghosh, Controlled doping in graphene monolayers by trapping organic molecules at the graphene-Substrate interface, ACS Appl. mater. Interfaces 9 (2017) 5375-5381. 